(C) 2017 IEEE. Personal use of this material is permitted. Permission from IEEE must be obtained for all other uses, in any current or future media, including reprinting/republishing this material for advertising or promotional purposes, creating new collective works, for resale or redistribution to servers or lists, or reuse of any copyrighted component of this work in other works. 


\title{
A Survey and Comparison Of Device-To-Device Architecture Using LTE unlicensed Band
}

\author{
Bushra Ismaiel $^{*}$, Mehran Abolhasan ${ }^{\dagger}$, David Smith ${ }^{\ddagger}$, Wei Ni ${ }^{\S}$, Daniel Franklin ${ }^{\ddagger}$ \\ * School of Computing and Communications Faculty of Engineering and Information Technology (FEIT), \\ University of Technology, Sydney, Australia \\ bushra.ismaiel@student.uts.edu.au, ${ }^{\dagger}$ mehran.abolhasan@uts.edu.au \\ $\dagger$ david.smith@data61.csiro.au, $§$ Wei.Ni@csiro.au \\ $\dagger$ daniel.franklin@uts.edu.au
}

\begin{abstract}
Due to the rapid increase in data traffic, one of the solutions provided by mobile operators is to operate Long Term Evolution (LTE) in the unlicensed $5 \mathrm{GHz}$ band, as the licensed spectrum is becoming scarce. Mobile operators can expand their network capacity by operating LTE in the unlicensed band at lower cost when compared with using other licensed bands. Device to Device (D2D) communication, proven to be another effective way to enhance the capacity of a network, enables direct data exchange of localized traffic of users in proximity. Applying D2D communication to LTE unlicensed $5 \mathrm{GHz}$ band will further improve the network performance and user experience. In this article, we will discuss the new type of solutions that have been proposed for LTE operating in an unlicensed $5 \mathrm{GHz}$ band that includes; LTE-Unlicensed (LTE-U), LTE-License Assisted Access (LTE-LAA), LTE WiFi Link Aggregation (LWA), and MuLTEfire. We will discuss the important features along with their advantages and disadvantages and compare these technologies as well. We simulate LTE-LAA, LWA and MuLTEfire technologies in the presence of Wi-Fi hotspot and compare their results. Furthermore, we apply D2D communication to these technologies and from the results we conclude that MuLTEfire can increase the throughput drastically but network saturates quickly. Whereas, applying D2D communication with LWA is beneficial for a scalable network as it will not only increase the network throughput but will increase the network capacity as well.
\end{abstract}

Index Terms-Long Term Evolution (LTE), LTE Unlicensed (LTE-U), LTE-License Assisted Access (LTE-LAA), LTE WiFi Link Aggregation (LWA), MuLTEfire, Wi-Fi, Device to Device Communication (D2D).

\section{INTRODUCTION}

The usage of unlicensed spectrum has provided an excellent opportunity for the mobile operators to meet the data requirement of mobile users. Mobile operators can expand the network capacity and provide a better quality of service (QoS) by using LTE in unlicensed $5 \mathrm{GHz}$ band at a lower cost [1].

The concept of LTE in the unlicensed spectrum was introduced first time in the TV white space [2]. In this scheme frequency hopping and time hopping was used by LTE small cells in TV white space band to reduce interference between other devices in the band. Currently, the crucial issue for LTE networks to exploit $5 \mathrm{GHz}$ unlicensed spectrum is the coexistence problem with Wi-Fi technology [3]. LTE adopts scheduling based access technology in licensed band, whereas
Wi-Fi works on contention-based access mechanism and carrier sensing technology. The LTE unlicensed proposal has been a big concern for the Wi-Fi vendors and service providers, which have used $5 \mathrm{GHz}$ band for a long time and want to continue to do so without being unfairly penalized by the introduction of LTE [1].

Recently new types of LTE-Wi-Fi aggregation solutions have been proposed at the radio link, as well at the TCP link [4]. One is carrier Wi-Fi, in which network operators can deploy their own $\mathrm{Wi}-\mathrm{Fi}$ to offload traffic and reduce congestion [5]. LTE unlicensed (LTE-U), is the first version of LTE unlicensed which was initially proposed by Ericsson and Qualcomm in 2013, based on 3GPP release 10-12 [1]. LTE-U works on the mechanism of carrier sensing adaptive transmission (CSAT) and can only be used in China, USA, South Korea and India [6]. For worldwide deployment, LTE unlicensed needs to deploy Listen-Before-Talk (LBT) mechanism. LTE-Licensed Assisted Access (LTE-LAA) has been standardised by $3 \mathrm{GPP}$ in release 13 for downlink operation, which uses the mechanism of LBT and is the modified version of LTE-U [7]. It is believed that if LTE-LAA and Wi-Fi use the same spectrum, LTE-LAA will have more chance to dominate the spectrum and Wi-Fi devices will keep deferring to LTE-LAA transmissions [4]. Wi-Fi users will easily be interfered by LTE-LAA users as soon as LTE-LAA signal becomes stronger, hence degrading the performance of both networks. An alternative solution was proposed to LTE-U/LTE-LAA, called as LTE Wi-Fi link aggregation (LWA). A part of LWA is released by 3GPP in release 13 [8]. LWA increases the capacity by offloading some of the LTE traffic through Wi-Fi using CSMA protocol. Some researchers believe that LWA will perform better in terms of co-existence than LTE-U/LTE-LAA in the presence of Wi-Fi as it offloads the traffic using the same protocol as Wi-Fi, hence sharing the spectrum fairly [4]. Also Wi-Fi companies are much in favour of LWA as it boosts the LTE user data without affecting pre-existing Wi-Fi users. Alcatel-Lucent is collaborating with Qualcomm to demonstrate LWA at Mobile World Congress [9]. Currently, Qualcomm proposed another LTE based technology known as Multifire that solely operates in an unlicensed band and does not require an anchor in the licensed spectrum. [10]. 


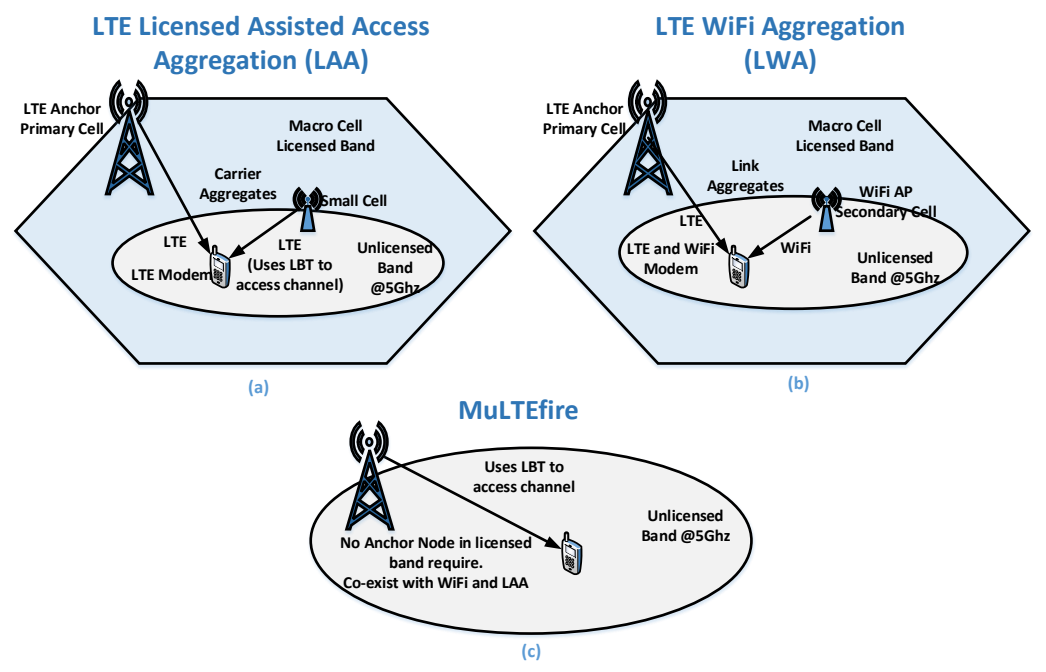

Fig. 1: LTE in unlicensed bands: LWA, LAA, MuLTEfire

Device to Device (D2D) communication an integral part of the future 5G standard, aims to improve the capacity, spectral efficiency, and coverage by enabling direct communication between the devices with minimal involvement from the base station (BS) in infrastructure assisted cellular system. D2D communication has been a central topic of research for quite some time and it has been shown that D2D communication increases the network throughput while minimizing the interference and power usage [11]. D2D communication can be very beneficial for the mobile operators when they combine D2D with LTE technologies operating in unlicensed $5 \mathrm{GHz}$ band.

Our main contribution in this paper is that we give a detail overview of LTE technologies operating in an unlicensed $5 \mathrm{GHz}$ band that includes; carrier Wi-Fi, LTE-U, LTE-LAA, LWA, and MuLTEfire. To the best of our knowledge, no paper has been published yet that discussed a detail overview of these technologies that includes; architecture, working mechanism, co-existence with Wi-Fi and overall cost. We also compare these technologies and conclude their advantages and disadvantages. Furthermore, we also present a detail simulation for LTE-LAA, LWA and MuLTEfire technologies in the presence of Wi-Fi hotspot and compare their results. We then investigate the performance of D2D communication over these strategies. It is concluded from the results that MuLTEfire can increase the throughput of users drastically but network saturates quickly. For a scalable network it will best to use D2D with LWA as it increases the throughput and can increase the capacity of the network as well.

\section{State Of ARt: LTE Networks Operating in UNLICENSED BANDS}

In this section, we will investigate the different solutions provided for LTE operating in an unlicensed $5 \mathrm{GHz}$ band.
We will discuss their architecture, working mechanism, cost and co-existence problem with Wi-Fi. Furthermore, we will compare these technologies with their advantages and disadvantages.

\section{A. Carrier Wi-Fi}

Carrier Wi-Fi is considered to be the first step for mobile operators to utilize the unlicensed bands. Network operators can deploy their own Wi-Fi access points (AP) to further offload traffic, increase coverage and reduce congestion.

Carrier Wi-Fi can lead to ineffectiveness of network management and low spectrum efficiency as it adopts different access and management mechanisms from LTE networks [5].

\section{B. LTE-Unlicensed (LTE-U)}

LTE-U uses the same LTE channels as LTE CA but unlike LTE CA LTE-U operates in unlicensed band at $5 \mathrm{GHz}$ [4]. In LTE-U, only LTE channel can work as the primary channel whereas the unlicensed channel can work as the secondary channel. LTE-U works on the mechanism of carrier sensing adaptive transmission (CSAT), where the channel is sensed by the LTE devices for a longer period of time (up to 200ms) and based on the activities define an ON and OFF duration for a duty cycle [5]. LTE devices can transmit when the duty cycle is in ON mode and remains silent when the duty cycle is in OFF mode. In the OFF mode, LTE-U allows the WiFi devices to access the system.

LTE-U requires a new installation of $5 \mathrm{GHz}$ LTE-enabled hardware on the device and small cells. LTE-U will only be implemented in the regions where regulation does not require LBT, such as China, Korea, India, and USA [6]. The biggest issue of LTE-U is its intrusion to Wi-Fi, as it can block the access to Wi-Fi users by giving priority to LTE-U users. 


\begin{tabular}{|c|c|c|c|c|c|}
\hline & Carrier Wi-Fi & LTE-U & LTE-LAA & LWA & MuLTEfire \\
\hline Features & $\begin{array}{l}\text { Deploy operator } \\
\text { own Wi-Fi }\end{array}$ & $\begin{array}{l}\text { LTE operates in } \\
\text { unlicensed } 5 \mathrm{GHz} \\
\text { band using CSAT }\end{array}$ & $\begin{array}{l}\text { LTE operates in } \\
\text { unlicensed } 5 \mathrm{GHz} \\
\text { band using LBT }\end{array}$ & $\begin{array}{l}\text { LTE operates in } \\
\text { unlicensed } 5 \mathrm{GHz} \\
\text { band aggregated } \\
\text { with carrier Wi-Fi }\end{array}$ & $\begin{array}{l}\text { LTE only } \\
\text { operates } \\
\text { in unlicensed } \\
5 \mathrm{GHz} \text { band } \\
\text { using LBT }\end{array}$ \\
\hline Advantage & Easy to deploy & $\begin{array}{l}\text { Unified } \\
\text { management } \\
\text { mechansim }\end{array}$ & $\begin{array}{l}\text { Unified management } \\
\text { mechanism. } \\
\text { Global standard. }\end{array}$ & $\begin{array}{l}\text { Easy to implement. } \\
\text { Quick for } \\
\text { commercialization }\end{array}$ & $\begin{array}{l}\text { Unified } \\
\text { management } \\
\text { mechansim in } \\
\text { unlicensed band }\end{array}$ \\
\hline Disadvantage & $\begin{array}{l}\text { Lack the } \\
\text { performance } \\
\text { benefits from } \\
\text { LTE in } \\
\text { unlicensed band }\end{array}$ & $\begin{array}{l}\text { Contention } \\
\text { problem with } \\
\text { WiFi. } \\
\text { Applicable } \\
\text { where CSAT } \\
\text { mechanism is } \\
\text { applied. No global } \\
\text { standardisation }\end{array}$ & $\begin{array}{l}\text { Contention problem } \\
\text { with Wi-Fi. } \\
\text { Commercialization } \\
\text { will take longer time }\end{array}$ & $\begin{array}{l}\text { For better QoS } \\
\text { latency } \\
\text { of the link between } \\
\text { the LTE eNB } \\
\text { and Wi-Fi AP } \\
\text { should be kept } \\
\text { low. }\end{array}$ & $\begin{array}{l}\text { Contention } \\
\text { Problem } \\
\text { with Wi-Fi. } \\
\text { Need to replace } \\
\text { or upgrade } \\
\text { old Wi-Fi AP } \\
\text { to get LTE } \\
\text { performance. }\end{array}$ \\
\hline $\begin{array}{l}\text { Access } \\
\text { Network } \\
\text { Cost }\end{array}$ & $\begin{array}{l}\text { Medium } \\
(\text { New Wi-Fi } \\
\text { AP) }\end{array}$ & $\begin{array}{l}\text { High (New LTE } \\
\text { enabled } \\
\text { cell with LTE-U) }\end{array}$ & $\begin{array}{l}\text { High (New LTE } \\
\text { enabled cell with } \\
\text { LTE-LAA) }\end{array}$ & $\begin{array}{l}\text { Medium (New } \\
\text { small Cell LWA } \\
\text { aware Wi-Fi AP) }\end{array}$ & $\begin{array}{l}\text { High (New } \\
\text { LTE enabled } \\
\text { Cell) }\end{array}$ \\
\hline $\begin{array}{l}\text { New Hardware } \\
\text { Support }\end{array}$ & No & $\begin{array}{l}\mathrm{UE} \text { and eNB } \\
(5 \mathrm{GHz})\end{array}$ & $\begin{array}{l}\text { UE and eNB } \\
(5 \mathrm{GHz})\end{array}$ & No & $\begin{array}{l}\text { UE and eNB } \\
(5 \mathrm{GHz})\end{array}$ \\
\hline $\begin{array}{l}\text { LTE-Wi-Fi } \\
\text { Coexistence } \\
\text { Problem } \\
\end{array}$ & No & $\begin{array}{l}\text { Contention } \\
\text { Problem }\end{array}$ & $\begin{array}{l}\text { Contention } \\
\text { Problem }\end{array}$ & No & $\begin{array}{l}\text { Contention } \\
\text { Problem }\end{array}$ \\
\hline Global Problem & No & $\begin{array}{l}\text { Global } \\
\text { Harmonization }\end{array}$ & Required & No & $\begin{array}{l}\text { Global } \\
\text { Harmonization } \\
\text { Required } \\
\end{array}$ \\
\hline Standardisation & 3GPP & $\begin{array}{l}\text { LTE-U } \\
\text { forum/3GPP }\end{array}$ & 3GPP & 3GPP & $3 \mathrm{GPP}$ \\
\hline
\end{tabular}

TABLE I: Comparison between Carrier Wi-Fi, LTE-U, LTE-LAA, LWA and MuLTEfire

\section{LTE-Licensed Assisted Access (LTE-LAA)}

LTE-LAA is the modified version of LTE-U and adopts LBT mechanism for the coexistence of LTE-LAA and Wi-Fi, as shown in Figure 1(a). In LBT, LTE device first listens to check for ongoing transmission. If the channel is clear it will transmit for a period of time and then back-off to re-check the channel availability, but if the channel is busy it will not transmit and keep on listening unless the channel is available [5]. The LTE device examine another channel if the current channel is busy after several attempts [5].

Two options for LBT schemes have been suggested by the European Union; Frame-Based Equipment (FBE) and Load-Based Equipment (LBE) [4]. In FBE,the transmit/receive structure is not directly demand-driven but has fixed timing. After every frame, clear channel assessment (CCA) is checked if the channel is free the data is transmitted otherwise it has to wait for another frame period. In LBE LBT, CCA is performed whenever there is data to transmit. If the channel is available data is transmitted but if the channel is busy it will retransmit the data after the back-off time during extended CCA (eCCA) [4].

LTE-LAA is developed with the single global solution framework and is replacing the current terminology LTE-U [12]. LTE-LAA is the version of LTE in an unlicensed band that 3GPP standardizes in Release 13 and is set to become a global standard as it strives to meet regulatory requirements worldwide [6]. As LTE-LAA is the modified version of LTE$\mathrm{U}$, it also requires an additional installation of $5 \mathrm{GHz}$ LTEenabled hardware.

The biggest concern for LTE-LAA is the co-existence with Wi-Fi technology. Qualcomm and Ericsson tested and claimed that LTE-LAA operates with little interference with Wi-Fi and can increase the system capacity with faster mobile broadband speed [13]. Whereas, some of the companies like Google are not in favour of LAA as it can block the access to Wi-Fi users and will degrade the overall network throughput of Wi-Fi by giving priority to LTE-LAA users [14].

\section{LTE WiFi Link Aggregation (LWA)}

LWA emerged as an alternative technology to LTE-U and LTE-LAA and is capable of leveraging existing Wi-Fi access points (AP) to improve the network performance [5]. LWA architecture is shown in Figure 1(b).

For the transmission of LTE traffic, LWA uses unlicensed band similar to LTE-U and LTE-LAA but the transmission is done through Wi-Fi. Unlike LTE-U/LAA, LWA does not require a new LBT based protocol, but LWA uses the current Wi-Fi protocol to transmit the LTE traffic. LWA base station performs scheduling of packets at PDCP layer and transmits some over LTE and other on Wi-Fi after encapsulating in Wi-Fi frames. All the packets received from LTE and Wi$\mathrm{Fi}$ are then aggregated at PDCP layer of LWA UE. LWA base stations can improve the LTE performance by managing the 
radio resources according to the load and RF conditions [4]. During this transmission, Wi-Fi APs can use LTE core network for authentication, security, billing, etc, without a dedicated Gateway (GW) and without disturbing the native Wi-Fi APs.

LWA uses LTE on LTE band and Wi-Fi on Wi-Fi band which is not the case in LTE-U/LTE-LAA. LWA requires the deployment of small cells and other WiFi APs near it can get a software upgrade to support LWA. The Wi-Fi APs can also support non-LWA traffic by using separate service set identifier (SSID) [5]. According to some researchers, LWA is a solution that leverage's the existing Wi-Fi APs and does not impact on an unlicensed band, hence improving the network performance [4].

\section{E. MuLTEfire}

MuLTEfire is based on 3GPP LTE-LAA and LTE-eLAA (LTE-enhanced licensed Assisted Access). Figure 1(c) shows the architecture for MuLTEfire. MuLTEfire performs like LTELAA and LTE-eLAA only the difference is that it operates in an unlicensed band without a licensed anchor node.

According to Qualcomm, MuLTEFire will benefit mobile operators with new deployment opportunities for offloading their mobile network traffic [10]. The primary goal for MuLTEfire is to provide a seamless user experience in a hyperdense network by combining the benefits of LTE technology with the simplicity of WiFi like deployments. It is a solution that may be attractive to network operators and cable operators that lack licensed spectrum, although this mode has not been discussed in 3GPP [6].

A comparison among the different LTE technologies operating in an unlicensed band; Carrier Wi-Fi, LTE-U, LTELAA, LWA, and MuLTEfire is shown in Table 1. It is easier to deploy carrier Wi-Fi as the network operator can easily deploy their own Wi-Fi to offload traffic but the spectrum efficiency cannot be same as of LTE as Wi-Fi and LTE works on different mechanism. LTE-U/LTE-LAA carrier aggregates the licensed and unlicensed band to further improve the performance, whereas LWA aggregates the link of licensed and unlicensed band and MuLTEfire solely operates in unlicensed band. The major disadvantage of LTE-U, LTE-LAA and MuLTEfire is the contention problem with Wi-Fi as compared to LWA. LTE-LAA and MuLTEfire are still in the process of getting standardised.

\section{Simulation}

In our simulation, we consider a single LTE macro cell with small cell deployed in it. This small cell is LTELAA/LWA/MuLTEfire, taking one at a time for simulation. We have also considered a single Wi-Fi AP deployed in the same LTE macro cell whose coverage area overlaps with the same small cell; LTE-LAA/LWA/MuLTEfire. The small cell (LTELAA/LWA/MuLTEfire) and Wi-Fi share the same $20 \mathrm{MHz}$ unlicensed sub-band within the $5 \mathrm{GHz}$ band. The number of Wi-Fi users are fixed and are associated with Wi-Fi AP. It is assumed that the number of LTE-LAA/LWA/MuLTEfire users are randomly distributed around the small cell. No handover are considered in this paper.

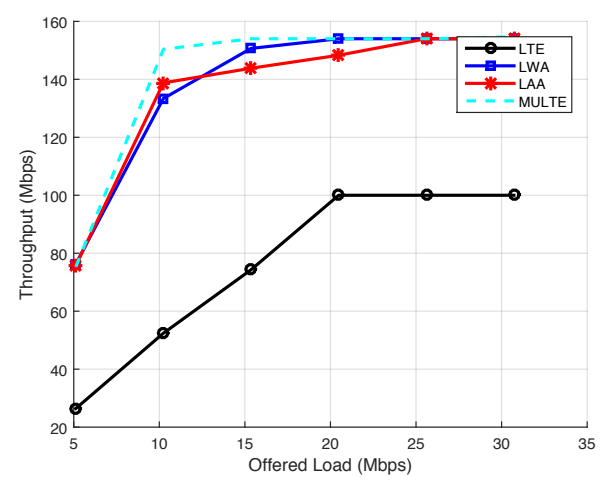

Fig. 2: Total Network Throughput vs Load

Throughput graph versus load is shown in Figure 2. MuLTEfire has higher throughput as it operates entirely in an unlicensed band using LBT and has more chance to capture the channel and transmit data. MuLTEfire saturates quickly as can be seen from the graph and might not be suitable for a scalable network as it can not accommodate more users. Whereas it can be observed from the graph that LWA can provide higher throughput when the load increases as compared to LTE-LAA. LTE-LAA and MuLTEfire can be a better solution for small loads as the throughput is higher whereas for higher load LWA can perform better.

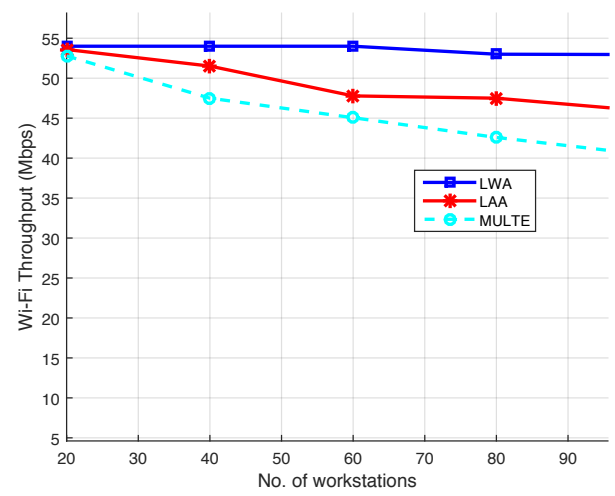

Fig. 3: Effect of Wi-Fi vs No of users

Figure 3 shows the effect of Wi-Fi users in the presence of a small cell; LTE-LAA, LWA, and MuLTEfire. The throughput of Wi-Fi users degrades in the presence of LTE-LAA small cell, as preference is given to LTE-LAA users over Wi-Fi users. This is because LTE has a continuously as well as a periodically transmitting protocol to transfer a variety of control and reference signals whereas $\mathrm{Wi}-\mathrm{Fi}$ is designed to coexist with other technologies through random backoff and channel sensing. Due to this Wi-Fi users will have little chance to sense a clear channel and transmit. Similarly, in case of MuLTEfire, the Wi-Fi throughput degrades further as it is LTE that is operating solely in an unlicensed band. Whereas, in 
case of LWA the Wi-Fi throughput is does not degrade much as there is no contention problem between the Wi-Fi and LWA users and can share the spectrum fairly.

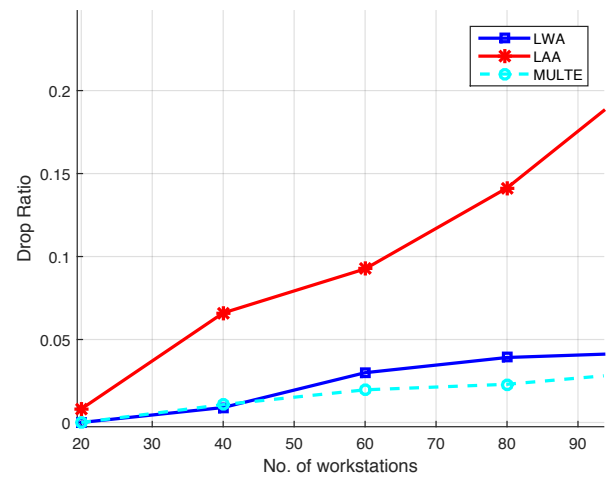

Fig. 4: Delay vs Number of workstation

Figure 4 illustrates the delay graph vs the number of users. It can be seen from the graph that LTE-LAA has higher delay than LWA. Due to the mechanism of LBT in LTE-LAA, it can increase the delay when contending for the channel in the presence of Wi-Fi. Whereas MuLTEfire has minimum delay as it solely operates in unlicensed band.

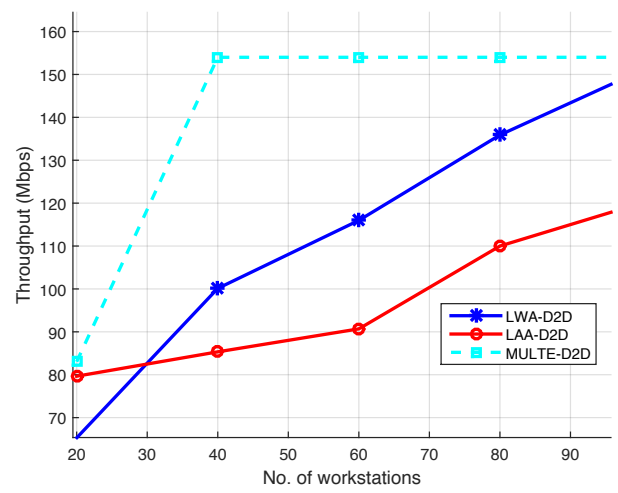

Fig. 5: Network Throughput with D2D communication

To further extend the idea we applied D2D communication in the given scenerio. We investigated the behaviour of D2D communication when applied to LTE-LAA, LWA and MuLTEfire. We observed from the Figure 5 that D2D communication in MuLTEfire can increase the throughput drastically as it operates only in unlicensed band and has more chance for the users to communicate through D2D communication. But it can also be observed that network saturates quickly when using D2D communication in MuLTEfire. Whereas, if we need to have a scalable network it will be more beneficial to apply D2D communication using LTE-LAA and LWA, as more users can be accomodated with higher throughput.

\section{CONCLUSION}

This paper presents a detailed overview of the solutions for LTE operating in unlicensed $5 \mathrm{GHz}$ band, along with their features and comparison. We have also simulated LTE-LAA, LWA, and MuLTEfire in the presence of Wi-Fi hot spot. From the simulation and results, we analyse that MuLTEfire can provide higher throughput but limit overall scalability of the network coexistence. Consequently, Wi-Fi users have a higher impact in the presence of MuLTEfire and can degrade the performance of Wi-Fi throughput. LTE-LAA has higher throughput for smaller load but the throughput decreases as the load increases and also Wi-Fi throughput degrades. Whereas LWA can provide better throughput than LTE-LAA for a large network and has minimal effect on the Wi-Fi users as there is no contention between the users. Similarly, when D2D communication is investigated in these techniques MuLTEfire can increase the throughput but cannot increase the users whereas LWA and LTE-LAA can accommodate more users, where LWA showing better throughput. LWA gives better results when a Wi-Fi AP is deployed near it and is more cost-effective and easy to implement as compared to LTELAA. For future challenges, it will be very important that all the technologies operating in $5 \mathrm{GHz}$ band should have equal control to access the medium to satisfy fairness. In future work, we plan to investigate the behaviour of D2D communication in these technologies using different scenarios in simulation and testbeds.

\section{REFERENCES}

[1] M. Paolini and S. Fili, "Lte unlicensed and wi-fi: Moving beyond coexistence."

[2] M. I. Rahman, A. Behravant, H. Koorapaty, J. Sachs, and K. Balachandran, "License-exempt lte systems for secondary spectrum usage: scenarios and first assessment," in New Frontiers in Dynamic Spectrum Access Networks (DySPAN), 2011 IEEE Symposium on. IEEE, 2011, pp. 349-358.

[3] H. Zhang, X. Chu, W. Guo, and S. Wang, "Coexistence of wi-fi and heterogeneous small cell networks sharing unlicensed spectrum," IEEE Communications Magazine, vol. 53, no. 3, pp. 158-164, 2015.

[4] Netmanias, "Analysis of LTE WiFi Aggregation Solutions," http: //www.netmanias.com/en/post/reports/8532/laa-lte-lte-u-lwa-mptcp/ analysis-of-lte-wifi-aggregation-solutions, 2016, [Online;2016].

[5] N. Zhang, S. Zhang, S. Wu, J. Ren, J. W. Mark, and X. S. Shen, "Beyond coexistence: Traffic steering in lte networks with unlicensed bands."

[6] B. Chen, J. Chen, Y. Gao, and J. Zhang, "Coexistence of lte-laa and wi-fi on $5 \mathrm{ghz}$ with corresponding deployment scenarios: A survey."

[7] 3GPP, "Analysis of LTE WiFi Aggregation Solutions," http://www.3gpp. org/news-events/3gpp-news/1789-laa_update, 2016, [Online;2016].

[8] — "Collaboration on LTE - WLAN Integration," http://www.3gpp. org/news-events/3gpp-news/1771-wlan_lte, 2016, [Online;2016].

[9] M. DeGrasse, "MWC15: Alcatel-Lucent combines Wi-Fi and cellular networks," http://www. rcrwireless.com/20150302/network-infrastructure/wi-fi/ alcatel-lucent-blends-wi-fi-and-cellular-tag4, 2015, [Online;2015].

[10] Qualcomm, "MulteFire: LTE-like performance with Wi-Fi-like deployment simplicity." https://www.qualcomm.com/invention/technologies/ lte/multefire, 2016, [Online;2016].

[11] K. Doppler, M. Rinne, C. Wijting, C. B. Ribeiro, and K. Hugl, "Deviceto-device communication as an underlay to lte-advanced networks," IEEE Communications Magazine, vol. 47, no. 12, pp. 42-49, 2009.

[12] R. Zhang, M. Wang, L. X. Cai, Z. Zheng, X. Shen, and L.-L. Xie, "Lteunlicensed: the future of spectrum aggregation for cellular networks," IEEE Wireless Communications, vol. 22, no. 3, pp. 150-159, 2015.

[13] Qualcomm, "Extending lte to unlicensed spectrum globally laa."

[14] R. Layton, "What The LTE-U Vs. WiFi Debate Is Really About," http://www.forbes.com/sites/roslynlayton/2015/09/02/ what-the-lte-u-vs-wifi-debate-is-really-about/\#538b71e74a03， 2015, [Online;2015]. 